Research Article

\title{
Do Environmental Regulations Improve Industrial Efficiency?
}

\author{
Peng Li $\mathbb{D}^{1,2}$ and Li-Li Shi ${ }^{1}$ \\ ${ }^{1}$ International Business School of Shaanxi Normal University, Xi'an 710119, China \\ ${ }^{2}$ School of Economics and Management, Northwest A\&F University, Yangling 712110, China \\ Correspondence should be addressed to Peng Li; hau_lipeng@snnu.edu.cn
}

Received 29 June 2021; Accepted 25 September 2021; Published 13 November 2021

Academic Editor: Guang-Liang Feng

Copyright (c) 2021 Peng Li and Li-Li Shi. This is an open access article distributed under the Creative Commons Attribution License, which permits unrestricted use, distribution, and reproduction in any medium, provided the original work is properly cited.

\begin{abstract}
This study was based on research on the impact of environmental regulation on industrial efficiency in 30 provinces from 2005 to 2017 in China. For the explained variables, the industrial efficiency of the DEA-Malmquist method was utilized for the decomposition and measurement of overall factor productivity, and government environmental governance variables were added as instrumental variables for two-stage least-squares regression. In addition, environmental regulatory intensity and year were utilized as threshold variables for the threshold test. In the benchmark regression, environmental regulation harms regional industrial efficiency. However, according to the IV estimation of government environmental governance variables, environmental regulation has a positive effect on the transformation of regional industrial efficiency. The influence of environmental regulation on industrial efficiency will be first suppressed and then promoted with the gradual increase of regulatory intensity. Furthermore, there is significant spatial heterogeneity in the impact of environmental regulations.
\end{abstract}

\section{Introduction}

Whether environmental regulation impedes industrial efficiency is somewhat controversial in academic circles. On the one hand, neoclassical research argues that environmental regulation raises production costs, raises unemployment, and reduces the competitiveness of economies. On the other hand, environmentalists and others argue that stronger environmental regulation policies can force firms to increase research and development of clean technologies, thereby reducing costs and environmental pollution. There are three theories in these mentioned studies. In the first place, there is the "Porter hypothesis-innovation compensation" effect, in which environmental regulation promotes innovation, which is generally based on overall factor productivity or its subtechnological progression. Second, there is the "double dividend" theory, in which environmental taxation will cause a "green dividend" of curbing pollution, improving the environment a "blue dividend" of increasing social employment, and promoting economic growth. Third, there is the "pollution sanctuary" effect, in which economic activities are relocated to areas with lax environmental regulations, resulting in the increased environmental pollution in the area in exchange for economic growth. The first two theories illustrate the positive effect of environmental regulation on industrial efficiency, while the last theory illustrates the inhibiting effect of environmental regulation on local green welfare. Since the "Porter hypothesis" [1] was proposed in 1991, a considerable number of scholars have started to verify whether environmental regulation affects firm productivity and innovation activities, but until now, the "Porter hypothesis" has not reached a consensus. Chakraborty and Chatterjee [2] constructed a nonequilibrium panel DID model using the German ban on azo dyes enacted in July 1994 as a quasi-natural experiment and found that the German dye ban had a significant effect on the innovation expenditure of upstream fuel manufacturing firms (upstream firms) in India. Trevlopoulos et al., Yang et al., and Zhang et al. explored the positive impact of environmental regulation on enterprise innovation and industrial structure upgrading [3-5]. Yu and Wang explained the economic benefit of environmental control policies in the process of industrial upgrading [6]. A considerable number of studies focus on the existence of the "pollution sanctuary effect", but 
there are some differences in the target population. Shi and $\mathrm{Xu}$ studied the impact of environmental regulations on import and export from the perspective of international trade flow [7]. Some scholars employed the flow of international capital as the object to verify whether the "pollution shelter effect" exists. For example, Lu and Wu utilized the Air pollution Prevention and Control Law to verify whether there is a pollution paradise effect [8]. Zhao et al. is the representative literature of studies on the pollution haven effect and the test of the double dividend hypothesis, who proposed the effect of environmental regulation on the spatial layout of industries and its effect through different geographic locations of polluting industries. Their evidence indicated that the water pollution industry migrates upstream after environmental regulation is enhanced [9]. Huang and Lei further studied how environmental regulation affects green investment in enterprises [10], while Stavropoulos et al. discussed the evolutionary relationship between environmental regulation and industrial competitiveness [11]. In addition, Zhang et al. made useful explorations from sustainable development, reusing industrial byproducts, and green construction in civil engineering [12-16], and further expanded the research scope of environmental regulation and industrial development.

In summary, these authors employed scientific research methods and instruments to scientifically determine the direction and extent of the effect of environmental regulation on industrial efficiency. The specific steps are as follows: first, according to the "Porter's hypothesis-innovation compensation" hypothesis, the DEA-Malmquist method is utilized to measure the industrial overall factor productivity (industrial TFP) index and its decomposition terms: efficiency change, technological progress, the industrial TFP index of 30 provinces, autonomous regions, and municipalities directly under the Central and Western regions of China from 2005 to 2017. Next, the commonly utilized composite index method was then employed to construct a comprehensive measurement system for the intensity of environmental regulations in Chinese industry to measure the intensity of environmental regulations. Third, the panel data of 30 Chinese provinces from 2005 to 2017 are selected, and the backward effect of environmental regulations on industrial efficiency is verified by using panel regression with fixed effects and the least-squares instrumental variables method [17]. Ultimately, through threshold regression [18], the threshold characteristics and spatial heterogeneity of environmental regulations are verified. Based on the spatial heterogeneity and related literature studies, foreign direct investment, regional economic development level, and provincial intervention policies are used as control variables to eliminate influencing factors besides the core explanatory variable of environmental regulation.

\section{The Measurement of Industrial Overall Factor Productivity}

2.1. Research Methodology-Malmquist Index. The Malmquist index has the advantage of further decomposing productivity changes into efficiency changes and technological progress. Among them, efficiency change refers to the change in organizational and managerial efficiency within the industry, which comes from the change in the efficiency of the original technology use and the efficiency change caused by the scale of production. Therefore, it can be further decomposed into pure technical efficiency change and scale efficiency change. Technological progress alludes to the productivity change caused by the introduction or upgrading of technology. This method has the advantages of not requiring factor price information and a specific form of a production function. It is effortless to calculate efficiency and can deal with multiple input and output problems, so it is more favored among scholars. Due to the limitation of space, this study briefly introduces the construction method of the Malmquist productivity index.

The Malmquist productivity index is defined using a distance function and refers to the geometric mean of the Malmquist productivity index in period $t$ versus period $t+1$. Assuming the premise that each provincial district is a decision unit, $\left(x^{t}, y^{t}\right)$ and $\left(x^{t+1}, y^{t+1}\right)$ represent the inputoutput quantities in period $t$ and period $t+1$, respectively, and the change in the input-output relationship means the change in productivity, denoted by $M_{i}^{t+1}$ :

$$
M_{i}^{t+1}\left(x^{t}, y^{t}, x^{t+1}, y^{t+1}\right)=\sqrt{\frac{D_{i}^{t}\left(x^{t}, y^{t}\right)}{D_{i}^{t}\left(x^{t+1}, y^{t+1}\right)} \times \frac{D_{i}^{t+1}\left(x^{t}, y^{t}\right)}{D_{i}^{t+1}\left(x^{t+1}, y^{t+1}\right)}} .
$$

In equation (1), $D_{i}^{t}\left(x^{t}, y^{t}\right)$ is the hybrid distance function, which is the inverse of the technical efficiency.

Productivity changes do not only come from technological progress but also efficiency changes. When we say efficiency, we mean the efficiency of utilizing the production technology, that is, the distance between the production frontier surface and the actual amount of output, denoted by $\mathrm{EFFCH}_{i}^{t+1}$; the technological progress is the movement of the production frontier surface, denoted by $\mathrm{TECHCH}_{i}^{t+1}$ :

$$
\begin{aligned}
\operatorname{TECHCH}_{i}^{t+1}\left(x^{t}, y^{t}, x^{t+1}, y^{t+1}\right) & =\sqrt{\frac{D_{i}^{t+1}\left(x^{t+1}, y^{t+1}\right)}{D_{i}^{t}\left(x^{t+1}, y^{t+1}\right)} \times \frac{D_{i}^{t+1}\left(x^{t}, y^{t}\right)}{D_{i}^{t+1}\left(x^{t+1}, y^{t+1}\right)}}, \\
\mathrm{EFFCH}_{i}^{t+1}\left(x^{t}, y^{t}, x^{t+1}, y^{t+1}\right) & =\frac{D_{i}^{t}\left(x^{t}, y^{t}\right)}{D_{i}^{t+1}\left(x^{t+1}, y^{t+1}\right)} .
\end{aligned}
$$

The Malmquist productivity index can be decomposed as the product of efficiency changes and technical progress:

$$
\begin{aligned}
M_{i}^{t+1}\left(x^{t}, y^{t}, x^{t+1}, y^{t+1}\right)= & \operatorname{EFFCH}_{i}^{t+1}\left(x^{t}, y^{t}, x^{t+1}, y^{t+1}\right) \\
& \times \operatorname{TECHCH}_{i}^{t+1}\left(x^{t}, y^{t}, x^{t+1}, y^{t+1}\right) .
\end{aligned}
$$

If $M>1$, it indicates overall productivity (TFP) growth; otherwise, it indicates a decline. If $\mathrm{EFFCH}>1$, it represents efficiency improvement, i.e., proper management style and decision-making; if EFFCH $<1$, it represents efficiency deterioration, i.e., improper management style and decision- 
making. If $\mathrm{TECHCH}>1$, it indicates an improvement in production technology; if $\mathrm{TECHCH}<1$, it indicates a decline in production technology.

\subsection{Input-Output Variables Selection and Data Sources.} Based on the availability of data and the need for empirical research, this study employed industrial enterprises above the scale to represent the whole industrial industry. In the research process, the overall industrial fixed assets of above-scale enterprises were utilized to replace capital input. In addition, the average annual number of all employees of industrial enterprises was utilized to replace labor input, and the industrial value-added of above-scale enterprises was selected as the expected output. The data were obtained from the China Statistical Yearbook and China Environmental Statistical Yearbook from 2005 to 2017.

2.3. Analysis of Measurement Results. This study employed DEAP2.1 software to calculate the industrial data of 30 provinces and regions in China from 2005 to 2017 to derive industrial TFP and its decomposition terms: efficiency change and technological progress.

Overall, China's industrial productivity from 2005 to 2017 generally indicated a slight fluctuation downward trend, which is chiefly due to the decline of the technical change index and is also in line with the current stage of China's economic adjustment into the "new normal" trend (Table 1). Since 2010, China's economic development has entered a period of growth rate shift, ending nearly 20 years of continuous economic growth. This can be seen as an indication that China's economy is being optimized and diversified. International experience indicates that when developing countries are in the development stage of economic start-up, they often pursue rapid economic growth and tend to neglect technological progress and structural optimization, resulting in problems like the imbalance between economic and social and urban and rural income distribution. As a result, the economy stagnates or even declines severely, falling into the "middleincome trap." In addition, a comparative study found that China's industrial overall factor productivity achieved positive growth in 2009-2010 and 2012-2013, which chiefly depended on the growth of the technological progress index.

As can be seen from Table 2, China's regional industrial TFP decreased in 2005-2017, with the eastern and western regions declining more significantly and the central region tending to be stable. Among the more economically developed provinces in the east of China, only Zhejiang Province has significantly improved its efficiency, and only Henan Province and Guizhou Province have improved their overall factor productivity in the central and western regions, but the efficiency of other provinces has not increased or declined. These all reflect that China's industrial enterprises are optimizing their industrial structure and seeking space for industrial upgrading.

\section{Selection, Measurement, and Analysis of Environmental Regulation Intensity Indicators}

3.1. Environmental Regulation Intensity Indicator (FERI). The implementation of environmental regulations depends on the willingness of local governments, the level of regional economic development, the current situation of environmental pollution, etc. Even if a country formulates a uniform regulatory policy, the intensity of implementation may vary from region to region. This study applied the comprehensive index method commonly utilized by scholars [8] to construct a comprehensive measurement system of the environmental regulation intensity of Chinese industries, including a target layer and four evaluation index layers (wastewater, waste gas, waste residue, and others) and calculate the environmental regulation intensity of individual pollutants and the comprehensive environmental regulation intensity of each province by assigning different weights to different pollutants. Based on the severity of various pollutant emissions in China and the availability of data, three individual indicators of wastewater treatment, waste gas, and solid waste investment in each province are selected to construct a comprehensive measurement system of environmental regulation intensity. This indicator construction method is as follows: in the first place, the three single indicators are linearly standardized; that is, the values of each indicator are converted to within the range of $[0,1]$ by mathematical transformations to eliminate the incommensurability between indicators and the contradiction between indicators. The calculation formula is as follows:

$$
P R_{i j}^{s}=\frac{P R_{i j}-\min \left(P R_{j}\right)}{\max \left(P R_{j}\right)-\min \left(P R_{j}\right)},
$$

where $i$ refers to the province $(i=1,2, \ldots 30)$ and $j$ refers to all types of pollutants $(j=1,2,3) ; P R_{i j}$ are the original values of each indicator, while $\max \left(P R_{j}\right)$ and $\min \left(P R_{j}\right)$ are the maximum and minimum values of the three individual indicators for each province each year. In addition, $P R_{i j}^{s}$ are the standardized values of each indicator.

The adjustment coefficient $\left(\omega_{i j}\right)$ and the weight are calculated for each indicator. For different provinces, the proportion of pollution emissions of "three wastes" varies greatly, so their treatment project investment also varies; for a particular province, the degree of treatment of different pollutant emissions also varies. Therefore, different weights are provided to the indicators of wastewater, waste gas, and solid waste in each province, and the weight of each indicator is adjusted to reflect the changes in the treatment of major pollutants in each province. The adjustment coefficients are calculated as follows:

$$
\omega_{i j}=\frac{E_{i j} / \sum E_{i j}}{Y_{i} / \sum Y_{i}}
$$

where $\omega_{i j}$ is the adjustment factor of pollutant $j$ in province $i$. $E_{i j}$ is the emission of pollutant $j$ in province $i$, so $\sum E_{i j}$ is the overall emission of the same pollutant in the country. 
Table 1: Industrial TFP index and its decomposition index from 2005 to 2017.

\begin{tabular}{|c|c|c|c|c|c|}
\hline Years & TEP changes & Efficiency changes & Technological advances & Pure technical efficiency & Scale efficiency \\
\hline $2006 / 2005$ & 1.015 & 1.043 & 1.000 & 1.016 & 1.059 \\
\hline $2007 / 2006$ & 1.002 & 1.091 & 0.987 & 1.015 & 1.094 \\
\hline $2008 / 2007$ & 0.988 & 1.184 & 0.993 & 0.995 & 1.170 \\
\hline $2009 / 2008$ & 0.955 & 0.972 & 0.965 & 0.990 & 0.929 \\
\hline $2010 / 2009$ & 1.112 & 1.018 & 1.073 & 1.037 & 1.132 \\
\hline $2011 / 2010$ & 0.980 & 1.158 & 0.990 & 0.990 & 1.135 \\
\hline $2012 / 2011$ & 0.998 & 0.986 & 0.992 & 1.006 & 0.984 \\
\hline $2013 / 2012$ & 1.009 & 0.916 & 1.020 & 0.988 & 0.923 \\
\hline $2014 / 2013$ & 0.977 & 1.013 & 0.984 & 0.993 & 0.989 \\
\hline $2015 / 2014$ & 0.946 & 0.990 & 0.964 & 0.982 & 0.936 \\
\hline $2016 / 2015$ & 1.001 & 1.010 & 0.995 & 1.006 & 1.010 \\
\hline $2017 / 2016$ & 0.983 & 1.099 & 0.978 & 1.005 & 1.081 \\
\hline 2005-2017 & 0.997 & 1.04 & 0.995 & 1.002 & 1.037 \\
\hline
\end{tabular}

TAвLE 2: Average values of industrial TFP indices and their decomposition indices in the three major regions, 2005-2017.

\begin{tabular}{|c|c|c|c|c|c|}
\hline Province & TEP changes & Efficiency changes & Technological advances & Pure technical efficiency & Scale efficiency \\
\hline Beijing & 0.992 & 1.022 & 1.000 & 0.992 & 1.015 \\
\hline Tianjin & 1.011 & 1.058 & 1.001 & 1.011 & 1.070 \\
\hline Hebei & 1.000 & 1.037 & 1.000 & 1.000 & 1.037 \\
\hline Liaoning & 0.989 & 1.050 & 0.983 & 1.006 & 1.039 \\
\hline Shanghai & 1.011 & 1.058 & 1.000 & 1.011 & 1.070 \\
\hline Jiangsu & 0.988 & 1.068 & 0.984 & 1.005 & 1.055 \\
\hline Zhejiang & 1.025 & 1.033 & 1.014 & 1.011 & 1.059 \\
\hline Fujian & 0.938 & 1.023 & 0.953 & 0.985 & 0.960 \\
\hline Shandong & 0.984 & 1.073 & 0.988 & 0.996 & 1.056 \\
\hline Guangdong & 0.991 & 1.067 & 1.000 & 0.991 & 1.057 \\
\hline Hainan & 1.006 & 1.041 & 1.008 & 0.998 & 1.046 \\
\hline Eastern region (mean) & 0.990 & 1.050 & 0.990 & 1.000 & 1.040 \\
\hline Shanxi & 1.020 & 1.031 & 1.015 & 1.004 & 1.051 \\
\hline Jilin & 1.002 & 1.022 & 1.002 & 1.000 & 1.024 \\
\hline Heilongjiang & 0.988 & 1.024 & 0.988 & 1.000 & 1.012 \\
\hline Anhui & 0.995 & 1.054 & 1.000 & 0.995 & 1.048 \\
\hline Jiangxi & 0.968 & 1.028 & 0.975 & 0.994 & 0.996 \\
\hline Henan & 1.043 & 1.041 & 1.038 & 1.004 & 1.085 \\
\hline Hubei & 1.007 & 1.024 & 1.006 & 1.002 & 1.032 \\
\hline Hunan & 1.000 & 1.036 & 1.000 & 1.000 & 1.036 \\
\hline Central region (mean) & 1.000 & 1.030 & 1.000 & 1.000 & 1.040 \\
\hline Neimenggu & 0.997 & 1.024 & 0.999 & 0.998 & 1.021 \\
\hline Guangxi & 0.994 & 1.022 & 1.000 & 0.994 & 1.016 \\
\hline Chongqing & 0.983 & 1.021 & 0.983 & 0.999 & 1.003 \\
\hline Sichuan & 0.993 & 1.027 & 0.994 & 1.000 & 1.020 \\
\hline Guizhou & 1.031 & 1.023 & 1.018 & 1.013 & 1.055 \\
\hline Yunnan & 0.959 & 1.028 & 0.959 & 0.999 & 0.986 \\
\hline Shanxi & 1.009 & 1.028 & 1.001 & 1.008 & 1.037 \\
\hline Gansu & 0.955 & 1.026 & 0.969 & 0.986 & 0.980 \\
\hline Qinghai & 1.015 & 1.071 & 1.000 & 1.015 & 1.088 \\
\hline Ningxia & 1.032 & 1.041 & 1.000 & 1.032 & 1.075 \\
\hline Xinjiang & 0.973 & 1.018 & 0.966 & 1.007 & 0.990 \\
\hline Western region (mean) & 0.990 & 1.030 & 0.990 & 1.000 & 1.020 \\
\hline
\end{tabular}

Moreover, $Y_{i}$ is the industrial value-added in province $i$, so $\sum Y_{i}$ is the national industrial value-added. After calculating the adjustment coefficients of exhaust gas, wastewater, and solid waste for each year, the average value of the adjustment coefficients during 2005-2017 was then calculated as $\bar{\omega}_{i j}$.
Ultimately, the standardized values and average weights of each indicator are utilized to calculate the intensity of environmental regulations in each province as

$$
\mathrm{FERI}_{i}=\frac{1}{3} \sum_{j=1}^{3} \bar{\omega}_{i j} \cdot P R_{i j}^{s} .
$$


The higher value indicates the more severe environmental regulation.

3.2. Analysis of Measurement Results. From Table 3, it can be seen that the average intensity of environmental regulations in the western region is significantly higher than that in the central and eastern regions, which laterally reflects that the industrial structure in the western region is significantly lower than that in the eastern and central regions. In the eastern region, the intensity of environmental regulations in Liaoning Province is significantly higher than that in other provinces, which is in line with the current industrial development of Liaoning Province as an old industrial production base whose urgent need is for industrial restructuring. In the central region, the environmental regulation intensity is higher in Anhui Province, and in the western region, the environmental regulation intensity is higher in Guizhou and Gansu Provinces, both of which possess a unique industrial structure and need to upgrade their industrial structures. In terms of years, from 2009, local governments in all provinces increased the intensity of environmental pollution control, which continued to a highintensity status in 2014. After 2015, the domestic industrial structure was significantly improved, and the intensity of environmental regulations was significantly moderated and improved.

\section{The Impact of Environmental Regulation Intensity on Industrial Efficiency: Model Setting and Transmission Mechanism Analysis}

\subsection{Setting of the Measurement Model}

4.1.1. Model Variable Design. To chiefly explore the impact of environmental regulation on industrial efficiency in China, a panel data regression method was utilized for empirical testing, and 30 provincial and urban administrative units across China from 2005 to 2017 were selected as the research objects. Formal environmental regulation indicators were incorporated into the measurement equation as the core explanatory variables, while other factors affecting industrial efficiency were introduced in the form of control variables. To prevent heterosexuality and multidisciplinary, the variables were logarithmically treated, and the specific variable design is shown in Table 4.

\subsubsection{Model Setting Test}

(1) Model 1. The cross-sectional individual variable coefficient model, or the variable coefficient model, is described in the following way:

$$
Y_{i t}=\alpha_{i}+X_{i t} \beta_{i}+\mu_{i t}, \quad i=1, \ldots, n, t=1, \ldots, T,
$$

where $X_{i t}$ is the $1 \times K$ vector, $\beta_{i}$ is the $1 \times K$ vector, and $K$ is the number of explanatory variables.
(2) Model 2. The cross-sectional individual variable-intercept model, or the variable-intercept model, is described as follows:

$$
Y_{i t}=\alpha_{i}+X_{i t} \beta+\mu_{i t}, \quad i=1, \ldots, n, t=1, \ldots, T .
$$

The model indicates that there are individual effects (variable intercepts) but no variation in the economic structure among cross-sectional individuals, so the structural parameters are the same across cross-sectional individuals.

(3) Model 3. The cross-sectional individual intercept, or coefficient invariant model, is described as follows:

$$
Y_{\mathrm{it}}=\alpha+X_{\mathrm{it}} \beta+\mu_{\mathrm{it}}, \quad i=1, \ldots, n, t=1, \ldots, T .
$$

The model indicates that there are no individual effects (variable intercepts) and no changes in economic structure among cross section individuals; therefore, both the intercept and structural parameters of the model are the same.

For all three models, it is tested whether the parameters inscribing the explanatory variables are constant at all crosssectional sample points and at a time; that is, it is tested in which of the previous three cases the problem under study falls to determine the form of the model. The widely utilized test is the covariance analysis test, also known as the F-test, which tests the following two main hypotheses.

Hypothesis 1. The slopes are the same at different crosssectional sample points and times, but the intercepts are not the same:

$$
H_{1}: y_{\mathrm{it}}=\alpha_{i}+X_{\mathrm{it}} \beta+\mu_{\mathrm{it}}
$$

Hypothesis 2. The intercept and slope are the same at different cross-sectional sample points and at a time:

$$
H_{2}: y_{\mathrm{it}}=\alpha+X_{\mathrm{it}} \beta+\mu_{\mathrm{it}} .
$$

To test the parametric constraint of the multiple linear regression model, if Hypothesis 2 is accepted, no further testing is required; if Hypothesis 2 is rejected, Hypothesis 1 should be tested to see if the slopes are all equal. If Hypothesis 1 is rejected, Model 1 should be utilized.

The results show that the hypothesis of "intercept and slope are the same across cross-sectional sample points and time" is rejected at the $5 \%$ significance level with $F=1.273$, while the hypothesis of "the slope is the same across crosssectional sample points and time, but intercept is different" is accepted at the $5 \%$ significance level with $F=1.293$ with different intercepts. Therefore, the fixed effects model should be chosen:

$$
\mathrm{IVA}_{\mathrm{it}}=\alpha_{i}+\mathrm{FERI}_{\mathrm{it}} \beta+\mathrm{FDI}_{\mathrm{it}} \gamma_{1}+\mathrm{GDP}_{\mathrm{it}} \gamma_{2}+\mathrm{EP}_{\mathrm{it}} \gamma_{3}+\mu_{i}+t_{t}+\varepsilon_{\mathrm{it}} .
$$

In the previous model, the corresponding parameters $\beta$, $\gamma_{1}, \gamma_{2}$, and $\gamma_{3}$ vary with cross-sectional individuals, where the explanatory variable is $\mathrm{IVA}_{\mathrm{it}}$ with the industrial valueadded of the region $i$ in year $t$, characterizing industrial efficiency. The core explanatory variable $\mathrm{FERI}_{\mathrm{it}}$ is the 
TABLE 3: Environmental regulation intensity in 30 Chinese provinces, 2005-2017.

\begin{tabular}{|c|c|c|c|c|c|c|c|c|c|c|c|c|c|c|}
\hline Region & 2017 & 2016 & 2015 & 2014 & 2013 & 2012 & 2011 & 2010 & 2009 & 2008 & 2007 & 2006 & 2005 & Mean \\
\hline Beijing & 0.04 & 0.04 & 0.04 & 0.01 & 0.02 & 0.02 & 0.02 & 0.02 & 0.03 & 0.09 & 0.07 & 0.29 & 0.25 & 0.07 \\
\hline Tianjin & 0.03 & 0.02 & 0.23 & 0.11 & 0.06 & 0.09 & 1.11 & 0.27 & 0.31 & 0.18 & 0.19 & 0.27 & 0.38 & 0.25 \\
\hline Hebei & 0.23 & 0.08 & 0.31 & 0.47 & 0.47 & 0.40 & 0.50 & 0.22 & 0.26 & 0.28 & 0.27 & 0.35 & 0.62 & 0.34 \\
\hline Liaoning & 1.03 & 0.34 & 0.72 & 0.88 & 2.82 & 2.27 & 1.47 & 3.84 & 2.80 & 2.80 & 1.76 & 2.51 & 0.44 & 1.82 \\
\hline Shanghai & 0.57 & 0.39 & 0.69 & 1.34 & 2.79 & 0.50 & 1.19 & 0.35 & 0.65 & 0.30 & 0.47 & 0.72 & 0.05 & 0.77 \\
\hline Jiangsu & 0.06 & 0.08 & 0.62 & 0.09 & 0.10 & 0.03 & 0.07 & 0.22 & 0.38 & 0.10 & 0.36 & 0.10 & 0.12 & 0.18 \\
\hline Zhejiang & 0.04 & 0.01 & 0.04 & 0.03 & 0.05 & 0.02 & 0.11 & 0.16 & 0.14 & 0.06 & 0.05 & 0.02 & 0.06 & 0.06 \\
\hline Fujian & 0.26 & 0.23 & 0.16 & 0.05 & 0.13 & 0.03 & 0.20 & 0.06 & 0.15 & 0.06 & 0.28 & 0.02 & 0.04 & 0.13 \\
\hline Shandong & 6.23 & 0.69 & 0.12 & 0.20 & 0.05 & 0.07 & 0.07 & 0.05 & 0.21 & 0.21 & 0.24 & 1.31 & 0.18 & 0.74 \\
\hline Guangdong & 0.41 & 0.30 & 0.28 & 0.12 & 0.51 & 0.43 & 0.23 & 0.15 & 0.21 & 0.18 & 0.54 & 0.26 & 0.45 & 0.31 \\
\hline Hainan & 0.18 & 0.24 & 0.29 & 0.18 & 0.30 & 0.24 & 0.07 & 0.07 & 0.34 & 0.05 & 0.06 & 0.13 & 0.05 & 0.17 \\
\hline Eastern region (mean) & 0.83 & 0.22 & 0.32 & 0.32 & 0.66 & 0.37 & 0.46 & 0.49 & 0.50 & 0.39 & 0.39 & 0.54 & 0.24 & 0.44 \\
\hline Shanxi & 0.15 & 0.28 & 0.10 & 0.05 & 0.13 & 0.18 & 0.06 & 0.07 & 0.15 & 0.13 & 0.11 & 0.12 & 0.22 & 0.13 \\
\hline Jilin & 0.05 & 0.31 & 0.33 & 0.20 & 0.35 & 0.18 & 0.15 & 0.31 & 0.21 & 0.10 & 0.11 & 0.51 & 0.96 & 0.29 \\
\hline Heilongjiang & 0.46 & 0.30 & 0.53 & 0.23 & 0.38 & 0.17 & 0.25 & 0.28 & 0.20 & 0.21 & 0.25 & 0.32 & 0.25 & 0.29 \\
\hline Anhui & 0.80 & 0.83 & 1.29 & 0.73 & 0.55 & 0.92 & 1.41 & 0.66 & 0.68 & 1.25 & 0.97 & 0.78 & 1.19 & 0.93 \\
\hline Jiangxi & 0.13 & 0.32 & 0.17 & 0.17 & 0.93 & 0.07 & 0.26 & 0.27 & 0.27 & 0.26 & 0.36 & 0.53 & 0.27 & 0.31 \\
\hline Henan & 0.08 & 3.42 & 0.05 & 0.06 & 0.11 & 0.10 & 0.10 & 1.28 & 0.98 & 0.13 & 0.17 & 0.52 & 0.23 & 0.56 \\
\hline Hubei & 0.06 & 0.05 & 0.08 & 0.07 & 0.20 & 0.26 & 0.15 & 0.53 & 0.24 & 0.17 & 0.39 & 0.61 & 0.42 & 0.25 \\
\hline Hunan & 0.06 & 0.04 & 0.12 & 0.04 & 0.07 & 0.22 & 0.06 & 0.09 & 0.09 & 0.02 & 0.21 & 0.20 & 0.19 & 0.11 \\
\hline Central region (mean) & 0.22 & 0.69 & 0.33 & 0.19 & 0.34 & 0.26 & 0.31 & 0.44 & 0.35 & 0.28 & 0.32 & 0.45 & 0.47 & 0.36 \\
\hline Neimenggu & 0.01 & 0.05 & 2.02 & 0.90 & 0.05 & 0.11 & 0.05 & 1.11 & 0.15 & 0.05 & 1.28 & 0.06 & 0.12 & 0.46 \\
\hline Guangxi & 0.06 & 0.01 & 0.04 & 0.04 & 0.03 & 0.06 & 0.05 & 0.07 & 0.06 & 0.02 & 0.03 & 0.08 & 0.00 & 0.04 \\
\hline Chongqing & 0.01 & 0.01 & 0.04 & 0.07 & 0.04 & 0.03 & 0.28 & 0.15 & 0.09 & 0.15 & 0.23 & 0.16 & 0.25 & 0.12 \\
\hline Sichuan & 0.05 & 0.02 & 0.04 & 0.06 & 0.08 & 0.08 & 0.20 & 0.08 & 0.13 & 0.26 & 0.26 & 0.45 & 0.34 & 0.16 \\
\hline Guizhou & 0.02 & 0.03 & 0.09 & 0.10 & 0.32 & 0.28 & 7.79 & 0.79 & 6.86 & 0.73 & 0.81 & 1.09 & 0.25 & 1.47 \\
\hline Yunnan & 0.04 & 0.15 & 0.58 & 5.36 & 0.86 & 0.73 & 0.58 & 0.99 & 0.88 & 1.62 & 0.37 & 0.55 & 0.15 & 0.99 \\
\hline Shanxi & 0.05 & 0.06 & 0.10 & 0.10 & 0.37 & 0.46 & 0.47 & 1.64 & 0.56 & 0.09 & 0.32 & 0.18 & 0.19 & 0.35 \\
\hline Gansu & 0.01 & 0.22 & 0.01 & 0.07 & 0.16 & 8.74 & 0.38 & 1.50 & 1.38 & 0.51 & 0.67 & 1.12 & 0.17 & 1.15 \\
\hline Qinghai & 0.05 & 0.22 & 0.59 & 0.36 & 0.26 & 0.04 & 0.22 & 0.40 & 0.23 & 0.02 & 0.01 & 0.01 & 0.00 & 0.19 \\
\hline Ningxia & 0.51 & 0.72 & 0.54 & 4.98 & 1.29 & 0.34 & 0.31 & 0.43 & 0.57 & 0.33 & 0.44 & 0.38 & 0.03 & 0.84 \\
\hline Xinjiang & 0.05 & 0.03 & 0.14 & 0.20 & 0.17 & 0.04 & 0.20 & 0.17 & 0.74 & 0.03 & 0.02 & 0.01 & 0.00 & 0.14 \\
\hline Western region (mean) & 0.08 & 0.14 & 0.38 & 1.11 & 0.33 & 0.99 & 0.96 & 0.67 & 1.06 & 0.35 & 0.40 & 0.37 & 0.34 & 0.54 \\
\hline Mean & 0.39 & 0.32 & 0.35 & 0.57 & 0.45 & 0.57 & 0.60 & 0.54 & 0.66 & 0.35 & 0.38 & 0.46 & 0.26 & \\
\hline
\end{tabular}

TABle 4: Model variable design.

\begin{tabular}{|c|c|c|c|}
\hline Variable type & Variable Name & Variable indicators & Variable representation \\
\hline \multirow{3}{*}{$\begin{array}{l}\text { Explained variables } \\
\text { Core explanatory } \\
\text { variables }\end{array}$} & Industrial efficiency & Industrial value-added & IVA \\
\hline & Environmental regulation & Environmental regulation intensity & FERI \\
\hline & Foreign direct investment & $\begin{array}{l}\text { Actual utilization of foreign direct investment (USD } \\
\text { million) }\end{array}$ & FDI \\
\hline \multirow[t]{2}{*}{ Control variables } & $\begin{array}{l}\text { Regional economic } \\
\text { development level }\end{array}$ & Overall GDP after deflating by region (billion yuan) & GDP \\
\hline & $\begin{array}{l}\text { Provincial intervention } \\
\text { policies }\end{array}$ & $\begin{array}{l}\text { The number of state-owned industrial enterprise units } \\
\text { above the scale (a) }\end{array}$ & $\mathrm{EP}$ \\
\hline
\end{tabular}

intensity of environmental regulations in region $i$ in year $t$. The control variables $\mathrm{FDI}_{\mathrm{it}}, \mathrm{GDP}_{\mathrm{it}}$, and $\mathrm{EP}_{\text {it }}$ are the actual amount of foreign direct investment utilized by the region $i$ in year $t$. The overall GDP of each region, the number of industrial enterprise units above the scale, characterizing foreign direct investment, the GDP of each region, and the intervention policy of each province are shown, respectively, in addition to controlling for individual fixed effects $\mu_{i}$ and time-fixed effects $t_{t}$.

Due to the association causality between the explanatory variables and the core explanatory variables, to mitigate their endogeneity, the authors employed a method of Chen and Chen [15] and selected the government environmental governance variable as an instrumental variable to measure the full picture of government environmental governance by the frequency of environment-related terms in provincial government work reports. This indicator not only measures the strength of local government environmental governance but also mitigates the endogeneity problems arising from the use of existing metrics.

To summarize, to quantitatively examine the impact of government environmental governance on industrial 
efficiency in China, the two-stage least-squares regression model (2SLS) is set as follows:

$$
\begin{aligned}
\mathrm{FERI}_{\mathrm{it}}= & \delta_{i} Z_{\mathrm{it}}+\mathrm{FDI}_{\mathrm{it}} \lambda_{1 i}+\mathrm{GDP}_{\mathrm{it}} \lambda_{2 i}+E P_{\mathrm{it}} \lambda_{3 i}+v_{\mathrm{it}}, \\
\mathrm{IVA}_{\mathrm{it}}= & \alpha_{i}+\mathrm{FERI}_{\mathrm{it}} \beta+\mathrm{FDI}_{\mathrm{it}} \gamma_{1}+\mathrm{GDP}_{\mathrm{it}} \gamma_{2} \\
& +\mathrm{EP}_{\mathrm{it}} \gamma_{3}+\mu_{i}+t_{t}+\varepsilon_{\mathrm{it}} .
\end{aligned}
$$

$Z_{\text {it }}$ is the number of environment-related terms, "environmental protection," "environmental protection," "pollution," "energy consumption," "emission reduction," "sewage," "ecology," "green," and the sum of the word frequencies of "low carbon." The previous 2SLS model is able not only to assess the impact of government environmental governance on environmental regulation but also to further screen the resulting impact on industrial efficiency.

\subsection{Analysis of the Impact of Environmental Regulation Intensity on Industrial Efficiency and Its Transmission Mechanism}

4.2.1. Fixed Effects Model Regression. The results of the fixed effects model estimation indicate that the coefficient of the environmental regulation variable is significantly negative, indicating that the increase in the intensity of environmental regulation has a significant negative impact on regional industrial efficiency. In other words, the more stringent government environmental protection measures and the higher demands on industrial enterprises will increase the operational pressure on enterprises, therefore reducing industrial efficiency. In contrast, the regression coefficients for foreign direct investment, gross regional product, and provincial intervention policies are all significantly positive and passed the $1 \%$ significance test, suggesting that these three factors contribute significantly to improving industrial efficiency (Table 5).

4.2.2. Regression Analysis of Instrumental Variables. Considering that the regression results of the baseline model are likely to have endogeneity problems, the frequency of words about environmental vocabulary in government work reports was utilized as an instrumental variable for environmental regulation, drawing on the approach of Chen Shiyi's scholar [6]. This indicator not only measures the strength of local government environmental governance but also mitigates the endogeneity problem of the model.

The parametric results of the instrumental variables regression indicate that the F-test values of the first stage regression, although small, are large enough to reject the initial hypothesis due to the large enough sample size, indicating that the selected instrumental variables are highly correlated with the endogenous variables. In the two-stage least-squares estimation model, the core explanatory variables of both Model 1 and Model 3 passed the 1\% significance test with positive coefficients, indicating that environmental regulation has a significant positive impact on industrial efficiency when instrumental variables are added for correction. The increased environmental protection efforts by the government have forced local industrial
TABle 5: Baseline model (fixed effects) estimation results.

\begin{tabular}{lcccc}
\hline \multirow{2}{*}{ Explanatory variables } & \multicolumn{4}{c}{ Explained variable: industrial efficiency } \\
& FE (1) & FE (2) & FE (3) & FE (4) \\
\hline $\begin{array}{l}\text { Environmental } \\
\text { regulation }\end{array}$ & -95.45 & -146.58 & -90.06 & -85.62 \\
$\begin{array}{l}\text { Foreign direct } \\
\text { investment }\end{array}$ & & $0.0029^{* * *}$ & $0.0009^{* * *}$ & $0.0007^{* * *}$ \\
$\begin{array}{l}\text { Gross regional product } \\
\begin{array}{l}\text { Provincial } \\
\text { intervention policies }\end{array}\end{array}$ & & $1.144^{* * *}$ & $1.154^{* * *}$ \\
$\begin{array}{l}\text { Individual fixed effects } \\
\text { Time-fixed effects }\end{array}$ & Yes & Yes & Yes & Yes \\
$\begin{array}{l}\text { Observations } \\
\text { Modified R-square }\end{array}$ & 390 & 390 & 390 & 390 \\
\hline
\end{tabular}

enterprises to transform and upgrade using technology, eliminating end-use industries and "screening" them, which greatly increases the proportion of green and high-tech enterprises and improves the industrial productivity of the whole region. Compared with the baseline regression, GDP and provincial intervention policies have a significant positive effect on the improvement of industrial efficiency. The higher the GDP is, the more favorable the construction of infrastructure and the introduction of a labor force is. Therefore, the more favorable the increase in industrial efficiency is, the higher the provincial intervention policy is. In other words, the higher the number of state-owned industrial units above the regional scale, the more conducive it is to uploading orders and implementing local government policies related to environmental protection. In the process of implementing environmental protection policies, subsidies will be provided for R\&D technology, which is more conducive to industrial efficiency (Table 6).

\subsection{Spatial Heterogeneity of Environmental Regulation and Industrial Efficiency}

4.3.1. Setting of the Panel Threshold Model. Empirical studies indicate that the backward effect of environmental regulation on industrial efficiency does exist, but the stronger the environmental regulation is, the more significant the backward effect on industrial efficiency is. When questioning the Porter hypothesis, Xu [19] pointed out that the Porter hypothesis is based on the premise of "properly designed environmental regulations." In a certain period, a country or region can withstand the impact of how many enterprises are eliminated at a certain limit. If more than a certain limit, such regulatory intensity and standards are not feasible. Too harsh or too relaxed regulatory intensity may not be conducive to the improvement of industrial efficiency. Therefore, there may be several "thresholds" for environmental regulations to affect industrial efficiency, and the impact on industrial efficiency may differ significantly depending on whether the relevant variables in each province cross the corresponding threshold. At the same time, considering the differences in the level of economic development, foreign investment, and the number of stateowned enterprises in each province, the relationship 
TABLE 6: Estimation results of instrumental variables (2SLS).

\begin{tabular}{|c|c|c|c|c|}
\hline \multirow{2}{*}{ Explanatory variables } & \multicolumn{4}{|c|}{ Explained variable: industrial efficiency } \\
\hline & IV_2SLS (1) & IV_2SLS (2) & IV_2SLS (3) & IV_2SLS (4) \\
\hline Environmental regulation & $14580.75^{* * *}$ & -34490.77 & $1605.082^{* * *}$ & 363.87 \\
\hline Foreign direct investment & & 0.0073 & $0.0003^{* * *}$ & 0.0006 \\
\hline Gross regional product & & & $1.312^{* * *}$ & $1.163^{* * *}$ \\
\hline Provincial intervention policies & & & & $0.086^{* * *}$ \\
\hline Phase I F-statistic & 3.16 & 3.10 & 3.04 & 2.96 \\
\hline Phase II F-statistic & 76.77 & 173.50 & 288.66 & 298.53 \\
\hline Observations & 390 & 390 & 390 & 390 \\
\hline Modified R-square & 0.28 & 0.32 & 0.74 & 0.97 \\
\hline
\end{tabular}

between the two may be spatial, indicating there may be spatial heterogeneity in the relationship between the two.

To empirically test this hypothesis, this study drew on Hansen [18], Wang [20], and other scholars to first endogenously group 30 Chinese provinces through threshold tests, followed by estimation and significance tests of the threshold characteristics of the environmental regulation effect for each subsample, which not only ensures the reliability of the threshold value but also enables examining the relationship between environmental regulation intensity and industrial efficiency in China "threshold effect." On this basis, a panel threshold regression was conducted to quantitatively analyze the spatial heterogeneity in the relationship between the two. The panel threshold regression model was set as follows:

$$
\begin{aligned}
\mathrm{IVA}_{\mathrm{it}}= & \alpha_{i}+\mathrm{FERI}_{\mathrm{it}} \beta_{1} \times d\left(q \leq \omega_{i}\right)+\mathrm{FDI}_{\mathrm{it}} \gamma_{1} \times d\left(q>\omega_{i}\right) \\
& +\mathrm{GDP}_{\mathrm{it}} \gamma_{2}+\mathrm{EP}_{\mathrm{it}} \gamma_{3}+\mu_{i}+t_{t}+\varepsilon_{\mathrm{it}},
\end{aligned}
$$

where $d(*)$ is the equation sex function, $q$ denotes the threshold variable, and $\omega_{i}$ is the specific threshold value; $\beta_{1}$ and $\beta_{2}$ denote the elasticity coefficients of the backward industrial efficiency of environmental regulation intensity at $q \leq \omega_{i}$ and $q>\omega_{i}$, respectively. The sign or estimates of $\beta_{1}$ and $\beta_{2}$ should be significantly different if the threshold choice is reasonable and the threshold estimates passed the significance.

4.3.2. Selection and Testing of Threshold Variables. According to the principle of the threshold regression model, it is known that the threshold variables can be either explanatory variables or other independent variables in the model. The intensity of environmental regulation and the level of economic development differ greatly among provinces, so the intensity of environmental regulation and its lag period, as well as the GDP of each province and its lag period, were selected as alternative threshold variables (Table 7).

Based on the threshold value, the 30 provinces are divided into three groups: "weakly regulated," "moderately regulated," and "strongly regulated" (Table 8). For the "weakly regulated" and "medium regulated" groups, they include the major economically developed provinces in China, like Guangdong, Zhejiang, Shanghai, Jiangsu,
Beijing, and other eastern provinces, which not only have a higher degree of marketization by their location advantages and economic policy inclination but also have a higher degree of their location advantages and economic policies. These provinces not only have a higher degree of marketization but also can optimize resource allocation and guide industrial restructuring with the help of market forces, therefore improving industrial efficiency. Moreover, the higher level of economic development makes these provinces more capable of introducing advanced foreign technology, high-end equipment, and advanced management experience. Consequently, the improvement of industrial efficiency relies more on the adjustment of factor input structure, upgrading, and technology than on environmental regulation. On the other hand, the implementation of environmental regulations is bound to cause increased costs for enterprises and at a given stage of economic development. There is always a limit to the ability of enterprises to bear rising costs, so the intensity of environmental regulations should be increased within a certain limit. Therefore, we cannot blindly increase regulation without causing the extinguishing of many enterprises, especially small- and medium-sized ones, because too much regulation leads to too a few enterprises.

"Strong regulation" has a positive backward elasticity coefficient; that is to say, the intensity of environmental regulation has a significant positive driving effect on industrial efficiency. In this group, typical industrial and resource-based provinces, like Jilin, Anhui, and Yunnan, are dominant. Based on the unique natural resource endowments of these provinces, primary industries like extractive industries, raw material processing industries, and traditional heavy industries with high energy consumption and pollution occupy an important position in economic development, forming a path to dependence on the "environment-for-growth" development model and an energy-driven development model. As the implementation and intensity of environmental regulations increase, severe environmental constraints are imposed on the existing industrial pattern of this group of provinces to establish an effective push-back mechanism, which not only helps this group of provinces prevent the dilemma of "resource curse" but also provides a strong driving force to break away from the established industrial structure and industrial pattern.

According to the threshold regression results, the backward effect of environmental regulation intensity on 
TABLE 7: Threshold variable selection and self-sampling test.

\begin{tabular}{lccccc}
\hline \multirow{2}{*}{ Threshold variable } & \multicolumn{2}{c}{ Single threshold } & \multicolumn{2}{c}{ Double threshold } & \multicolumn{2}{c}{ Triple threshold } \\
& F-statistic & $P$ value & F-statistic & $P$ value & $F$-statistic \\
\hline Feri & 14.25 & $0.0233^{*}$ & 6.95 & 0.2833 & 8.27 \\
Feri(-1) & 52.34 & $0.0033^{* *}$ & 41.15 & $0.0467^{* *}$ & 34.13 \\
GDP & 213.13 & 0.2400 & 7.34 & 0.2400 & 0.4267 \\
GDP(-1) & 211.13 & $0.0001^{* * *}$ & 5.24 & 0.4267 & 0.6267 \\
\hline
\end{tabular}

TABLE 8: Results of sample grouping based on threshold values.

\begin{tabular}{lcc}
\hline Subgroups & $\begin{array}{c}\text { Threshold variable } \\
\text { values }\end{array}$ & Provinces included in each group \\
\hline $\begin{array}{l}\text { Weak } \\
\text { regulation }\end{array}$ & Feri $(-1) \leq 0.69$ & $\begin{array}{c}\text { Beijing, Hebei, Zhejiang, Liaoning, Fujian, Henan, Guangdong, Shanxi, } \\
\text { Heilongjiang, Hubei, Hunan, Guangxi, Chongqing, Sichuan, Qinghai, Xinjiang, } \\
\text { Shaanxi }\end{array}$ \\
$\begin{array}{l}\text { Medium } \\
\text { regulation } \\
\begin{array}{l}\text { Strongly } \\
\text { regulated }\end{array}\end{array}$ & $0.69<$ Feri $(-1) \leq 1.38$ & Shanghai, Inner Mongolia, Tianjin, Jiangsu, Hainan \\
\hline
\end{tabular}

TABLE 9: Panel threshold regression results.

\begin{tabular}{|c|c|c|c|}
\hline Explanatory variables & Parameter estimates & OLS standard error & $t$ value \\
\hline Foreign direct investment & $-0.309^{* *}$ & -0.136 & -2.27 \\
\hline Regional economic development level & 0.309 & 0.304 & 1.02 \\
\hline Provincial intervention policies & -0.201 & -0.165 & -1.22 \\
\hline Feri $(-1) \leq 0.69$ & -3.567 & $1.388^{* *}$ & -2.57 \\
\hline $0.69<$ Feri $(-1) \leq 1.38$ & -0.112 & $0.048^{* *}$ & -2.32 \\
\hline $1.38 \leq$ Feri $(-1)$ & 1.362 & 1.221 & -1.12 \\
\hline
\end{tabular}

industrial efficiency is neither monotonically increasing nor decreasing, and the backward elasticity coefficient of environmental regulation intensity varies significantly across provinces. That is to say, as the intensity of regulation changes from weak to strong, it will have an impact on industrial efficiency that is first inhibited and then promoted. When the industrial value-added is higher than 1.38 units, each unit increase in environmental regulation intensity will promote the regional industrial value-added to 1.362 units. When the environmental regulation intensity crosses the second threshold value of 0.69 , the backward elasticity coefficient decreases from 1.362 to -0.112 and passes the $5 \%$ significance test. As the threshold variable crosses the threshold value of 0.69 , the backward elasticity coefficient decreases further, from -0.112 to -3.567 , and this result still passes the $5 \%$ significance test (Table 9).

\section{Discussion and Conclusion}

At present, China's economic development has shifted toward a "new normal," the goal of which is to establish sustainable economic growth through establishing a symmetrical economic structure emphasizing the structure of economic growth rather than the overall economy, such as greening industrial efficiency adjustment. Using panel data of 30 Chinese provinces from 2005 to 2017, this study employed panel regression of fixed effects and the leastsquares instrumental variables method to test whether environmental regulation can promote industrial efficiency and the push-back mechanism and verified the threshold characteristics and spatial heterogeneity of environmental regulation through threshold regression. This study indicated environmental regulation would effectively improve industrial production efficiency. As different industries have different marginal cost functions when environmental regulations impose severe environmental constraints on emitting enterprises, enterprises with lower marginal costs gain comparative advantages in "green" development, while enterprises with higher marginal costs will gradually shrink in size due to their poor ability to bear rising costs. Severe formal environmental regulations enable the elimination of pollution-intensive backward and overcapacity, therefore creating a driving force for industrial efficiency improvement. Next, this study discovered 30 Chinese provinces could be classified into "strong," "medium," and "weak" regulations according to whether the intensity of environmental regulations crosses the corresponding threshold. It was found that the majority of Chinese provinces fall into the "weak regulation" group. Further threshold regressions indicated that the impact of environmental regulation on industrial efficiency shows a significant threshold characteristic. That is to say, as the intensity of regulation gradually increases, it will have a suppressive and then promotional impact on industrial efficiency, as well as producing significant spatial heterogeneity. 
With the findings of related studies, the following policy insights are obtained. On the one hand, differentiated environmental regulation policies should be developed. To fully exploit and utilize the potential of environmental regulation to improve industrial efficiency, it is necessary to consider the pollution characteristics of different provinces and the spatial heterogeneity of environmental regulation to push-back industrial efficiency and to develop differentiated regulatory policies and intensity. It is not appropriate to raise environmental regulations in provinces where they do not facilitate industrial restructuring. Environmental controls should also be relaxed, relying more on the innovation of industrial policies and the resource allocation and competitive functions of the market, deepening the reform of market mechanisms, and taking full advantage of the market's power to promote dynamic industrial restructuring. For provinces where environmental regulation can effectively improve industrial efficiency, environmental regulation should be utilized as a driving force to establish a long-term mechanism by using higher flexibility of pushback. Local governments should appropriately increase the intensity of existing regulations, set stricter environmental standards, and improve the exit mechanism of enterprises to fundamentally curb resource-depleting production patterns and traditional path dependence, therefore contributing to a sustainable and clean industrial structure. Within a certain period, the increase in the intensity of environmental regulations must be limited to what enterprises can afford because promoting industrial efficiency through environmental constraints is a long-term process that cannot be achieved overnight but should be designed gradually and on a rolling basis based on what enterprises can afford.

On the other hand, we should promote reliance on technology and optimize resource allocation through the market. With the gradual increase of environmental awareness in the public mind, people now have a conscious preference for "technology," "clean," and "environmental protection" products. Therefore, as a producer of industrial enterprises, it should also be time to seize the public's favorite taste, production of environmentally friendly products, and then use technology to differentiate production. This is also a reverse behavior to guide consumers to green consumption. When designing environmental regulation policies, the government should not only restrict the production and emission behavior of producers but also consider consumer behavior and influence resource allocation by guiding and encouraging "green" consumption, thereby forming a new path for industrial transformation.

\section{Data Availability}

The data used to support the findings of this study are all included within the article.

\section{Conflicts of Interest}

The authors declare that they have no conflicts of interest.

\section{Acknowledgments}

This work was supported by the National Natural Science Foundation of China Youth Project (71703082), Postdoctoral Fund (2017M613233), and Shaanxi Normal University Central University Basic Scientific Research Business Fund Special Fund Project (20200347). The authors thank TopEdit (https://www.topeditsci.com) for its linguistic assistance during the preparation of this manuscript.

\section{References}

[1] M. E. Porter and C. V. D. Linde, "Toward a new conception of the environment-competitiveness relationship," The Journal of Economic Perspectives, vol. 9, no. 4, pp. 97-118, 1995.

[2] P. Chakraborty and C. Chatterjee, "Does environmental regulation indirectly induce upstream innovation? New evidence from India," Research Policy, vol. 46, no. 5, pp. 935-955, 2017.

[3] N. S. Trevlopoulos, T. A. Tsalis, K. I. Evangelinos, K. P. Tsagarakis, K. I. Vatalis, and I. E. Nikolaou, "The influence of environmental regulations on business innovation, intellectual capital, environmental and economic performance," Environment Systems and Decisions, vol. 41, no. 1, pp. 163-178, 2021.

[4] L. Yang, J. Zhang, and Y. Zhang, "Environmental regulations and corporate green innovation in China: the role of city leaders' promotion pressure," International Journal of Environmental Research and Public Health, vol. 18, no. 15, 2021.

[5] G. Zhang, P. Zhang, Z. G. Zhang, and J. Li, "Impact of environmental regulations on industrial structure upgrading: an empirical study on the Beijing-Tianjin-Hebei region in China," Journal of Cleaner Production, vol. 238, Article ID 117848, 2019.

[6] X. Yu and P. Wang, "Economic effects analysis of environmental regulation policy in the process of industrial structure upgrading: evidence from Chinese provincial panel data," The Science of the Total Environment, vol. 753, Article ID 142004, 2021.

[7] X. Shi and Z. Xu, "Environmental regulation and firm exports: evidence from the eleventh five-year plan in China," Journal of Environmental Economics and Management, vol. 89, no. 89, pp. 187-200, 2018.

[8] Y. Lu and L. Wu, Is There a Pollution Haven Effect? Evidence from a Natural Experiment in China, Mimeo, N Y. USA, 2012.

[9] C. Zhao, E. Kahn, Y. Liu et al., "The consequences of spatially differentiated water pollution regulation in China," Journal of Environmental Economics and Management, vol. 88, pp. $468-485,2018$.

[10] L. Huang and Z. Lei, "How environmental regulation affect corporate green investment: evidence from China," Journal of Cleaner Production, vol. 279, Article ID 123560, 2021.

[11] S. Stavropoulos, R. Wall, and Y. Xu, "Environmental regulations and industrial competitiveness: evidence from China," Applied Economics, vol. 50, no. 12, pp. 1378-1394, 2018.

[12] T. Zhang, Y. L. Yang, and S. Y. Liu, "Application of biomass by-product lignin stabilized soils as sustainable Geomaterials: a review," The Science of the Total Environment, vol. 728, Article ID 138830, 2020.

[13] T. Zhang, G. Cai, and S. Liu, "Application of lignin-based byproduct stabilized silty soil in highway subgrade: a field investigation," Journal of Cleaner Production, vol. 142, pp. 4243-4257, 2017. 
[14] G. L. Feng, B. R. Chen, Q. Jiang, Y. X. Xiao, W. J. Niu, and P. X. Li, "Excavation-induced microseismicity and rockburst occurrence: similarities and differences between deep parallel tunnels with alternating soft-hard strata," Journal of Central South University, vol. 28, no. 2, pp. 582-594, 2021.

[15] T. Zhang, G.-J. Cai, and S.-Yu Liu, "Application of ligninstabilized silty soil in highway subgrade: a macroscale laboratory study," Journal of Materials in Civil Engineering, vol. 30, no. 4, Article ID 04018034, 2018.

[16] G. L. Feng, X. T. Feng, B. R. Chen, Y. X. Xiao, and Y. Yu, "A microseismic method for dynamic warning of rockburst development processes in tunnels," Rock Mechanics and Rock Engineering, vol. 48, no. 5, pp. 2061-2076, 2015.

[17] S. Xu, "Environmental regulation and firm competitiveness: a challenge based on Porter's hypothesis," Journal of International Trade, vol. 32, no. 5, pp. 78-83, 2010.

[18] B. E. Hansen, "Sample splitting and threshold estimation," Econometrica, vol. 68, no. 3, pp. 575-603, 2000.

[19] S. Chen and D. Chen, "Haze pollution, government governance, and high-quality economic development," Economic Research, vol. 53, no. 02, pp. 20-34, 2018.

[20] Q. Wang, "Fixed-effect panel threshold model using s," STATA Journal: Promoting communications on statistics and Stata, vol. 15, no. 1, pp. 121-134, 2015. 